\title{
Usabilidade de Interfaces Responsivas para m-Learning
}

\author{
Flávia L. Arantes ${ }^{1}$, Diovan C. de Queiroz ${ }^{1}$, André C. da Silva ${ }^{1,2}$, Fernanda M. P. \\ Freire $^{1}$
}

${ }^{1}$ Núcleo de Informática Aplicada à Educação (NIED) - Universidade Estadual de Campinas (UNICAMP)

Rua Seis de Agosto, 50 - Cidade Universitária - Campinas - SP - Brazil

${ }^{2}$ Instituto Federal de São Paulo (IFSP) - Campus Hortolândia

Av. Thereza Ana Cecon Breda, s/n, Vila São Pedro - Hortolândia - SP - Brazil

\author{
farantes@unicamp.br, fnstar21@gmail.com, \\ andre.constantinodifsp.edu.br, ffreiredunicamp.br
}

\begin{abstract}
Frameworks with responsive resources have become popular for web applications developing, so the same pages that serve for desktops and for mobile devices. However, the automatic adjustment of interfaces introduces interaction problems. The objective of this research is to conduct usability studies of responsive interfaces for m-learning and to propose adaptations of the interfaces when appropriate.
\end{abstract}

Resumo. Frameworks com recursos responsivos têm se tornado populares para o desenvolvimento de aplicações web, com isso as mesmas páginas servem para desktops e para dispositivos móveis. Entretanto, a adaptação automática das interfaces introduz problemas de interação. O objetivo desta pesquisa é realizar estudos de usabilidade de interfaces responsivas para mlearning e propor adaptações das interfaces, quando necessário.

\section{Introdução}

O uso de tecnologias nas escolas, em especial dos dispositivos móveis, é uma tendência mundial. Uma pesquisa da OECD (Organisation for Economic Co-operation and Development $)^{1}$ mostra que cada vez mais as escolas optam pelos dispositivos móveis, pelo fato de oferecerem maior flexibilidade de uso quando comparados aos desktops.

Um levantamento divulgado pela Exame.com² em outubro de 2014, mostra que no Brasil, $78 \%$ dos jovens usam smartphone e $42 \%$ possuem tablet, números comparáveis aos da Europa Ocidental e EUA e que superam o uso de PCs (69\%). Com tamanha popularidade dos dispositivos móveis, os ambientes de e-learning estão construindo soluções para oferecer acesso a suas plataformas via dispositivos móveis, o que chamamos de m-learning.

As soluções de m-learning dividem-se em 3 categorias: i) aplicativo específico para dispositivo, ii) web site específico para dispositivos móveis e iii) web site que serve

\footnotetext{
${ }^{1}$ A OECD é uma organização formada por 34 países (http://www.oecd.org/about/membersandpartners/). Os dados da pesquisa são referentes aos dispositivos da própria escola, sem considerar os dispositivos pessoais dos alunos. Acesso em 23/05/2017.

${ }^{2}$ http://exame.abril.com.br/tecnologia/78-dos-jovens-usam-smartphone-mostra-pesquisa/. Acesso em 23/05/2017.
} 
para desktops e dispositivos móveis [Silva et al., 2013]. Essa última solução considera o uso de interfaces responsivas [Marcotte, 2011], as quais são adaptadas de acordo com o tamanho da tela do dispositivo. Com o aumento do poder de processamento dos dispositivos móveis, essa solução tem ganhado espaço, pois é necessário renderizar as páginas web e fazer as adaptações necessárias no cliente. A grande vantagem é ter apenas um código para fazer a manutenção. Entretanto, a adaptação das interfaces nos dispositivos móveis leva a alguns problemas de usabilidade que precisam ser contornados para que as interfaces não apresentem problemas de interação nos diferentes dispositivos.

Atualmente, estamos em fase de prototipação do TelEduc 5, que usa tecnologias com recursos responsivos para seu desenvolvimento. Considerando a complexidade envolvida no projeto de interfaces responsivas, nosso objetivo é realizar estudos de usabilidade das interfaces do TelEduc 5 quando acessadas por meio de tablets e smartphones, com vistas a encontrar soluções adequadas tanto para os dispositivos móveis quanto para desktops.

\section{Metodologia}

A metodologia desta pesquisa está relacionada à realização das seguintes atividades:

a) Desenvolvimento das interfaces do TelEduc 5 utilizando tecnologias com recursos responsivos.

b) Elaboração de um plano de testes de usabilidade para smartphones e tablets, considerando as interfaces responsivas desenvolvidas em a).

c) Aplicação do plano de testes e identificação de problemas de usabilidade.

d) Implementação de soluções para contornar os problemas de usabilidade identificados em c).

e) Testes com usuários considerando cenários de m-learning.

\section{Resultados Parciais}

Frameworks com recursos de responsividade tem se tornado populares para o desenvolvimento de aplicações web. As interfaces do TelEduc 5 estão em desenvolvimento utilizando os frameworks Bootstrap ${ }^{3}$ e Angular ${ }^{4}$, os quais oferecem recursos para implementação de interfaces responsivas. A Figura 1(a) mostra o protótipo da página de entrada da ferramenta Atividades, na tela de um desktop. A Figura 1(b) mostra a mesma interface na tela do smartphone Galaxy S5.

$\mathrm{Na}$ adaptação dessa e de outras interfaces para dispositivos móveis, duas situações podem acontecer: ajustes feitos automaticamente pelos frameworks e ajustes feitos pelo programador. No exemplo da Figura, os seguintes ajustes foram feitos:

- Ajustes automáticos: o ajuste das interfaces para caber a descrição das atividades e o menu tab na tela do celular foi feito pelos próprios frameworks responsivos, sem a necessidade de código adicional por parte dos programadores. O "colapse" da busca também foi feito pelos frameworks, com isso, a caixa de texto para digitar a busca

\footnotetext{
${ }^{3} \mathrm{https}: / / \mathrm{v} 4$-alpha.getbootstrap.com/

${ }^{4}$ https://angular.io/
} 
VI Congresso Brasileiro de Informática na Educação (CBIE 2017)

Anais do XXVIII Simpósio Brasileiro de Informática na Educação (SBIE 2017)

aparece no celular quando o usuário clica no ícone da lupa, enquanto no desktop, ambos já aparecem na interface.

- Ajustes feitos pelo programador: o logotipo do TelEduc ficou grande para a versão mobile, por isso o programador escreveu um código que escolhe um logotipo menor se o acesso for feito por um dispositivo com tela pequena. $\mathrm{O}$ menu para criação de atividades também precisou de código adicional para ficar adequado. Nesse caso, ao clicar no ícone dos três pontinhos ( $\vdots$ ) o menu aparece. Outros ajustes ainda precisam ser feitos no código, por exemplo, o menu que está à esquerda da versão desktop não aparece na versão mobile e o nome do curso não aparece por completo. É importante observar que a maior parte dos ajustes feitos pelo programador são facilitados pelo fato dos frameworks serem responsivos.

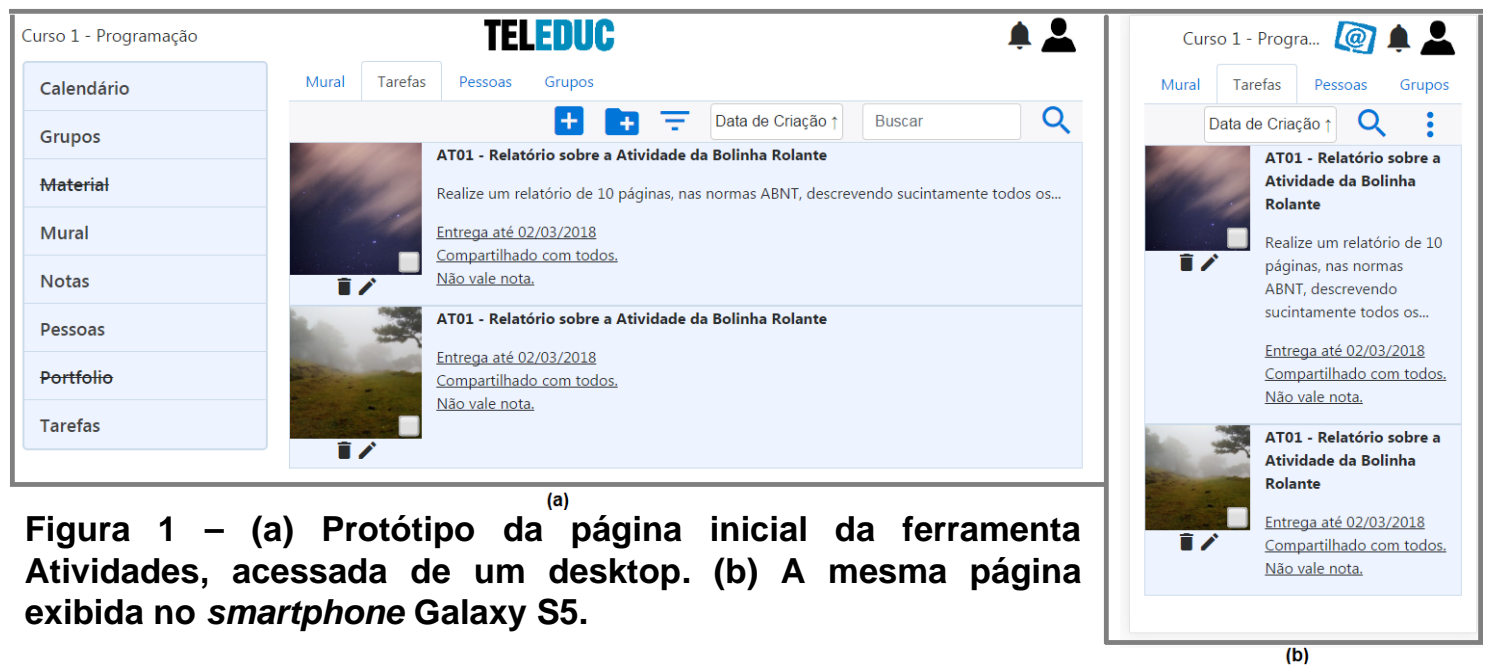

\section{Considerações Finais}

Os resultados parciais nos motivam a manter as tecnologias adotadas, Bootstrap e Angular, para dar continuidade à implementação das interfaces responsivas do TelEduc 5. Embora esforços adicionais sejam exigidos, grande parte do trabalho do programador é facilitada pelos frameworks.

Nossos trabalhos futuros são a elaboração de planos de testes de usabilidade para dispositivos móveis, a aplicação dos testes e a implementação dos ajustes necessários. Em seguida serão realizados testes com usuários visando avaliar a versão mobile em cenários de ensino-aprendizagem.

\section{Agradecimento}

Agradecemos ao apoio do CNPq por meio do projeto no 462478/2014-9.

\section{Referências}

Marcotte, E. (2011) “Responsive web design”. A Book Apart (Ed.), 2011. Ebook disponível em https://abookapart.com/products/responsive-web-design. Acesso em 22/05/2017.

Silva, A. C; Freire, F. M. P.; Rocha, H. V. (2013) "Identifying Cross-Platform and CrossModality Interaction Problems in e-Learning Environments". In: ACHI $2013-6^{\text {th }}$ International Conference on Advances in Computer-Human Interactions, 2013, Nice/França. Proceedings of ACHI 2013, v. 1. p. 243-249. 